TAIWANESE JOURNAL OF MATHEMATICS

Vol. 3, No. 3, pp. 339-356, September 1999

\title{
A NONCONFORMING WEAK RESIDUAL ERROR ESTIMATOR FOR ELLIPTIC PARTIAL DIFFERENTIAL EQUATIONS*
}

\author{
Jang Jou and Jinn-Liang Liu
}

\begin{abstract}
A nonconforming weak residual error estimator is presented and analyzed for finite element solutions of linear elliptic partial differential equations. The treatment of the flux jumps across element edges is of special interest. The estimator is obtained by solving local residual problems which do not explicitly involve the jumps and do not require boundary conditions. The estimator handles both interior and edge residuals on each element by a suitable construction of the basis functions for the local problems. Together with the previous conforming estimator, the weak residual error estimation, without the flux jumps, can thus be applied to both odd- and even-order finite element approximations.
\end{abstract}

\section{INTRODUCTION}

Weak residual error estimators are shown to be very effective for a large class of variational problems $[1,2,8,9,11,13-19,21]$. The formula used to derive these estimators can be generically expressed as follows: In each finite element $\tau_{i}$, determine $\tilde{e}_{i} \in S_{i}^{c}$ such that

$$
B\left(\tilde{e}_{i}, v_{i}\right)=F\left(v_{i}\right)-B\left(u_{h}, v_{i}\right) \quad \forall v_{i} \in S_{i}^{c},
$$

where $B(\cdot, \cdot)$ and $F(\cdot)$ are the bilinear and linear forms associated with a given variational problem, $u_{h} \in H$ is its finite element solution in some Sobolev space $H$, and $S_{i}^{c}$ is a finite element space defined over that element.

Received October 27, 1997; revised August 20, 1998.

Communicated by W.-W. Lin.

1991 Mathematics Subject Classification: 65N15, 65N30.

Key words and phrases: Adaptivity, finite element, a posteriori error estimate.

* This work was supported by NSC under grant 85-2121-M-009-014, Taiwan. 
An error estimator is said to be conforming if $S_{i}^{c} \subset H$ and nonconforming if $S_{i}^{c} \not \subset H$. The estimators of $[1,2,8,9,11,14,19]$ belong to the conforming type. Nonconforming estimators can be found in $[15,18]$.

Two important properties of the estimators based on formula (1.1) should be emphasized. First, the (weak) residual term of (1.1) does not explicitly include any flux jumps (the normal derivatives of $u_{h}$ ) across element edges. The treatment of the jumps is one of the major concerns of other estimators in which the jump terms are explicitly involved and/or specific local boundary conditions are imposed for the local problems (see, e.g., [3, 4, 5-8, 10, 20$21,23,24]$ ). Second, the performance of the weak residual error estimators is strongly influenced by the construction of the subspace $S_{i}^{c}$ which in turn depends on the finite element order of the approximate solution $u_{h}$. It is wellknown [7] that for second-order elliptic problems the discretization error of odd-order finite element solutions is mainly due to the jumps, while that of even-order approximations occurs principally in the interior of the elements. Therefore, the conforming estimators will fail for odd-order approximations if the support of the basis functions of $S_{i}^{c}$ does not cross over element edges [15]. This excludes many important classes of approximations, for example, the linear approximations. On the other hand, if the support does cross over element edges, (1.1) will result in a global system which obviously is not efficient for adaptive computations.

The purpose of this article is to give a more specific treatment of the jumps than that of [15] and [18]. The treatment is based on a particular construction of $S_{i}^{c}$ which is essentially an extension of the midpoint shape functions of [10] to the weak residual formulation (1.1). Under the treatment, a new formulation of (1.1) is given and the corresponding error estimator is proved to be equivalent to the exact error in the $H^{1}$ semi-norm for linear self-adjoint elliptic partial differential equations.

The main idea of the new formulation is to localize the left-hand side of (1.1) while conforming test functions are used for the right-hand side in order to estimate both the edge and the interior errors. More specifically, formula (1.1) is transformed to the determination of $\tilde{e}_{i} \in S_{i}^{c}$ such that

$$
B_{h}\left(\tilde{e}_{i}, v_{i}\right)=\frac{1}{2}\left(F\left(\hat{v}_{i}\right)-B\left(u_{h}, \hat{v}_{i}\right)\right) \quad \forall v_{i} \in S_{i}^{c}
$$

where $S_{i}^{c} \not \subset H$ but $\hat{v}_{i} \in H$. The conforming shape functions $\hat{v}_{i}$ are defined by extending the nonconforming functions $v_{i}$ from the element $\tau_{i}$ to its neighbors. The "nonconforming" bilinear form $B_{h}$ is a restriction of the conforming bilinear form $B$ to each element. The formulation is first presented for Poisson's equation in Section 2. In this case, the bilinear form $B(\cdot, \cdot)$ corresponds to the 
Laplace operator. In Section 3, formula (1.2) is changed to

$$
B_{h}\left(\tilde{e}_{i}, v_{i}\right)=\frac{1}{2}\left(F\left(\hat{v}_{i}\right)-A\left(u_{h}, \hat{v}_{i}\right)\right) \quad \forall v_{i} \in S_{i}^{c},
$$

where the bilinear form $A(\cdot, \cdot)$ corresponds to the more general elliptic operator. A numerical example is given in Section 4.

We briefly remark on the merit of using estimators based on formula (1.3). Although the model problems considered here are of elliptic PDEs, the same principle of the error estimation can be applied to more general problems and more general numerical methods if one refers to the formula in terms of the general bilinear $B(\cdot, \cdot)$ and linear $F(\cdot)$ forms. For example, this approach has been used for finite volume $[15,17]$, least squares finite element $[13,17]$, and boundary element [16] computations and for various boundary value problems such as parametrized nonlinear problems [15, 19], PDEs of mixed type [14, 15], semiconductor device simulation model [17], obstacle and free boundary value problems [15,17], advection-diffusion problems [17], and the Navier-Stokes equations $[13,16,17]$ etc.. The most important features of the present error estimation are unification, generality, and simplification. In other words, the principal formula used to derive the estimators for either different numerical methods or various boundary value problems is the same. Moreover, it allows spectral orders to vary in each element since the local space $S_{i}^{c}$ can be constructed by using the next higher-order hierachical shape functions [22] to that of $u_{h}$ in that element. Consequently, a combination of the conforming estimator of $[9,11]$ and the present noncoforming estimator is suitable for general $p$ or $h p$-version finite element methods. The implementation of the estimators is straightforward since formula (1.3) is almost identical to that of approximation, i.e., $A\left(u_{h}, v_{h}\right)=F\left(v_{h}\right)$. However, we must stress that the approach is not necessarily more efficient than other estimators for typical problems or for certain approximations since (1.3) always results in a solution of local problems whereas some estimators may only require calculation of residual terms or postprocess of higher-order terms (cf. [3, 5, 7, 20, 23, 24]).

\section{An Error Estimator for Poisson's Equation}

Let $\Omega$ be a bounded region in the plane with a Lipschitz boundary $\partial \Omega=$ $\partial \Omega_{D} \cup \partial \Omega_{N}$, where $\partial \Omega_{D}$ is a nonempty subset of $\partial \Omega$. For any open subset $\tau$ of $\Omega$, we denote by $H^{1}(\tau)$ the usual Sobolev space equipped with the semi-norm $|\cdot|_{1, \tau}$. For simplicity, we write $|\cdot|_{1}=|\cdot|_{1, \Omega}$. Consider the boundary value problem

$$
\begin{aligned}
-\Delta u(x) & =f(x) & & \text { in } \Omega, \\
u & =0 & & \text { on } \partial \Omega_{D}, \\
\frac{\partial u}{\partial n} & =g & & \text { on } \partial \Omega_{N} .
\end{aligned}
$$


The associated variational problem is to find $u \in H(\Omega)$ such that

$$
B(u, v)=F(v) \quad \forall v \in H(\Omega),
$$

where

$$
\begin{aligned}
H(\Omega) & :=\left\{u \in H^{1}(\Omega): u=0 \text { on } \partial \Omega_{D}\right\}, \\
B(u, v) & :=\int_{\Omega} \nabla u \cdot \nabla v d x, \\
F(v) & :=\int_{\Omega} f v d x+\int_{\partial \Omega_{N}} g v d s .
\end{aligned}
$$

For $v \in H(\Omega)$, define the (energy) $B$-norm

$$
\|v\|_{B}:=\sqrt{B(v, v)}=|v|_{1}
$$

and its restriction to any subset $\tau$ of $\Omega$

$$
\|v\|_{B, \tau}=|v|_{1, \tau}:=\left(\int_{\tau} \nabla v \cdot \nabla v d x\right)^{1 / 2} .
$$

To discretize (2.2), we introduce a finite-dimensional subspace $S \subset H(\Omega)$, which is associated with a mesh $T_{h}=\left\{\tau_{i} \mid i=1,2, \cdots, M\right\}$ on $\bar{\Omega}$. The mesh is characterized by a mesh size $h$. For any two distinct elements (triangles or rectangles or both) $\tau_{i}$ and $\tau_{j}$ in $T_{h}, \tau_{i} \cap \tau_{j}$ is either empty, a single vertex, or a common edge. Two elements are said to be adjacent if they have a common edge. For a given rectangular element, let $h_{\max }$ and $h_{\min }$ denote the largest and smallest edge lengths, respectively. Then the element edge ratio is defined by $h_{\min } / h_{\max }$. We always assume that the mesh $T_{h}$ belongs to a regular family of meshes on $\bar{\Omega}$. Recall that, see, e.g., [4,8], the family is regular if all angles of its triangular elements and all edge ratios of rectangular elements are bounded below by some constant $\sigma>0$. Shape regularity does not require a mesh to be globally quasi-uniform, but it does imply local quasi-uniformity of the mesh; an important property that will be exploited below.

The Lax-Milgram theorem asserts that there is a unique solution $u_{h} \in S$ satisfying the equation

$$
B\left(u_{h}, v_{h}\right)=F\left(v_{h}\right) \quad \forall v_{h} \in S .
$$

Let $e=u-u_{h}$ denote the exact error of the approximate solution $u_{h}$. Substituting $u=u_{h}+e$ into problem (2.2), we have

$$
B(e, v)=F(v)-B\left(u_{h}, v\right) \quad \forall v \in H(\Omega) .
$$


Weak residual error estimators are derived by some sort of approximation of the error equation (2.4). The approximation is based on an enlarged subspace $\bar{S} \subset H(\Omega)$ defined by

$$
\bar{S}=S \oplus S^{c}, \quad S \cap S^{c}=\{0\}, \quad S \neq\{0\}, \quad S^{c} \neq\{0\} .
$$

Here $S^{c}$ is a complementary space of $S$ in $\bar{S}$ and preserves the conformity. Hence there exists a unique function $u_{\bar{h}} \in \bar{S}$ satisfying

$$
B\left(u_{\bar{h}}, v\right)=F(v) \quad \forall v \in \bar{S} .
$$

In general, $u_{\bar{h}}$ is a better approximation of $u$ than $u_{h}$. In error analysis, this observation is frequently expressed as the following saturation assumption.

Assumption 1. The finite element solutions $u_{h}$ and $u_{\bar{h}}$ of (2.3) and (2.6) satisfy the inequality

$$
\left\|u-u_{\bar{h}}\right\|_{B} \leq \rho\left\|u-u_{h}\right\|_{B},
$$

where $\rho \in[0,1)$ is a constant independent of the mesh size $h$.

Let $\gamma=\sup \left\{B(w, v)\left\|w \in S_{h},\right\| w\left\|_{B}=1, v \in S_{h}^{c},\right\| v \|_{B}=1\right\}$. Then, obviously, we have $\gamma<1$ by the definition of the space $S^{c}$ in (2.5). However, it is not clear that the constant $\gamma$ is uniformly independent of the mesh parameter $h$ for all adaptive meshes. It is shown, for example, in [8] and [12] that this is indeed the case for the inner product induced by the bilinear form that corresponds to the general elliptic PDEs.

Lemma 1. There exists a constant $\gamma \in[0,1)$ independent of $h$ such that

$$
|B(w, v)| \leq \gamma\|w\|_{B}\|v\|_{B} \forall w \in S, \forall v \in S^{c} .
$$

Conforming error estimators are obtained by solving the conforming error problem: Determine $e^{c} \in S^{c}$ such that

$$
B\left(e^{c}, v\right)=F(v)-B\left(u_{h}, v\right) \quad \forall v \in S^{c} .
$$

The following result is well-known (see $[8,9,11,14,18]$ ).

Theorem 1. Let $u \in H(\Omega)$ and $u_{h} \in S$ be the solutions of (2.2) and (2.3), respectively, and $e=u-u_{h}$. If Assumption 1 holds for $S$ and $\bar{S}$, then (2.9) has a unique solution $e^{c} \in S^{c}$ and

$$
(1-\rho) \sqrt{1-\gamma^{2}}\|e\|_{B} \leq\left\|e^{c}\right\|_{B} \leq\|e\|_{B},
$$


where $\gamma \in[0,1)$ and $\rho \in[0,1)$ are independent of $h$.

In view of the bilinear form $B(\cdot, \cdot)$, the equation $(2.9)$ can be considered as a general formula that leads to various error estimators for a large class of variational problems in which the problem (2.1) is only a special case (see $[1,2,9,11,13-19])$. A general framework of this type of estimators cast in an abstract variational setting is presented in [15]. However, the conforming complementary subspace $S^{c}$ in (2.9) would result in a global system of linear equations if its individual basis functions have supports on more than one element. On the other hand, if the basis functions have supports only on their individual elements, the resulting error estimator will not be effective for oddorder finite element solutions $u_{h}$ since, for such a case, the major errors occur on the edges of elements [7].

Many estimators have been proposed to handle these errors (flux jumps) across elements (see e.g. [3, 5-8, 10, 20,21, 23, 24]). All those estimators involve the jumps. We propose here another way to treat the jumps. The objective of the treatment is to retain the weak residual term of (2.9) without explicitly involving the jumps. The resulting error estimator handles the jumps in a more specific way than that of [15]. Moreover, assumptions required for the present estimator are weaker than that of [15]. The jumps are handled indirectly by a proper construction of the basis functions of the complementary space $S^{c}$. We require the conforming space $S^{c}$ to have a locally affine basis [11] and satisfy (2.5).

For simplicity, we assume that the approximation is linear, i.e., $S$ consists of piecewise linears. The following results hold for more general approximation with some technical modifications. We define

$$
S^{c}=\operatorname{span}\left\{\phi_{i j}\right\} \subset H(\Omega),
$$

where each $\phi_{i j}$ is a basis function with the nodal point at the center of the common edge of the adjacent pair $\tau_{i}$ and $\tau_{j}$ and with the support on $\tau_{i} \cup \tau_{j}$, for all $\tau_{i} \in T_{h}$. Note that $\phi_{j i}=\phi_{i j}$ for all the adjacent pairs. For each element $\tau_{i} \in T_{h}$ the restriction of $\phi_{i j}$ on $\tau_{i}$ can be identified with a shape function on a fixed reference triangle or rectangle $\hat{\tau}$ via the affine transformation which maps the reference element $\hat{\tau}$ one-to-one onto $\tau_{i}$. In particular, we may consider that $\phi_{i j}$ are side-mode shape functions of degree two (cf. [22]). The resulting enlarged space $\bar{S}$ will then be hierarchical.

We again assume that the enlarged space so constructed satisfies Assumption 1. Hence, the estimate (2.10) still holds for this particular conforming subspace. Note that, under definition (2.11), the conforming problem (2.9) results in a global system of equations. 
The next step is to localize (2.9) while keeping the (weak) residual form of (2.9) and not requiring local boundary conditions or the jumps. For each basis function $\phi_{i j} \in S^{c}$, we define

$$
\tilde{\phi}_{j}^{i}=\left\{\begin{aligned}
\phi_{i j}, & \text { on } \tau_{i}, \\
0, & \text { otherwise. }
\end{aligned}\right.
$$

Similarly,

$$
\tilde{\phi}_{i}^{j}=\left\{\begin{aligned}
\phi_{i j}, & \text { on } \tau_{j}, \\
0, & \text { otherwise. }
\end{aligned}\right.
$$

Note that the halved-functions (2.12) were first introduced in [8] for solving local residual problems with jumps. For each element $\tau_{i}$, let $K(i)$ denote an index set of $j \neq i$ such that $\tau_{j}$ is an adjacent element of $\tau_{i}$. Let $|K(i)|$ denote the number of halved-functions (2.12) constructed on $\tau_{i}$. We assume that $|K(i)| \geq 1$, i.e., the mesh $T_{h}$ contains at least two elements. Note that $|K(i)| \leq 3$ if $\tau_{i}$ is triangular and $|K(i)| \leq 4$ if $\tau_{i}$ is rectangular. Define

$$
S_{i}^{c}=\operatorname{span}\left\{\tilde{\phi}_{j}^{i} \mid j \in K(i)\right\} .
$$

It is obvious that $S_{i}^{c}$ are not contained in $H(\Omega)$ nor is their direct sum

$$
S^{n}=S_{1}^{c} \oplus S_{2}^{c} \oplus \cdots \oplus S_{M}^{c} .
$$

Since all functions in (2.13) vanish at the vertices of the mesh $T_{h}$ and are continuous in $\tau_{i},|\cdot|_{1, \tau_{i}}$ is a norm on $S_{i}^{c}$ and consequently the mapping

$$
v \mapsto\|v\|_{B}:=\left(\sum_{\tau_{i} \in T_{h}}\|v\|_{B, \tau_{i}}^{2}\right)^{\frac{1}{2}}
$$

is a norm for all $v \in S^{n}$.

The following result is an extended version of Lemma 3.3 of [11] to the nonconforming case.

Lemma 2. There exist two positive constants $C_{1}$ and $C_{2}$ independent of the mesh parameter $h$ such that

$$
C_{1} \sum_{k \in K(i)}\left\|d_{k}^{i} \tilde{\phi}_{k}^{i}\right\|_{B}^{2} \leq\left\|\tilde{v}_{i}\right\|_{B}^{2} \leq C_{2} \sum_{k \in K(i)}\left\|d_{k}^{i} \tilde{\phi}_{k}^{i}\right\|_{B}^{2}
$$

for each element $\tau_{i}, i=1,2, \cdots, M$, and for any $\tilde{v}_{i}=\sum_{k \in K(i)} d_{k}^{i} \tilde{\phi}_{k}^{i} \in S_{i}^{c}$.

Proof. We use the affine mapping $\theta_{\tau_{i}}: \hat{\tau} \mapsto \tau_{i}$ for each element $\tau_{i} \in T_{h}$ to define

$$
\hat{v}_{i}=\tilde{v}_{i} \circ \theta_{\tau_{i}}
$$


on the fixed reference element $\hat{\tau}$. Then

$$
\hat{v}_{i}=\sum_{k \in K(i)} d_{k}^{i} \tilde{\phi}_{k}^{i} \circ \theta_{\tau_{i}}=\sum_{k \in K(i)} d_{k}^{i} \hat{\phi}_{k}^{i}
$$

where $\hat{\phi}_{k}^{i}=\tilde{\phi}_{k}^{i} \circ \theta_{\tau_{i}}, k \in K(i)$. Each function $\hat{\phi}_{k}^{i}$ can be identified with a basis function in a finite-dimensional space $\hat{S}^{c}$ that is defined on the reference element $\hat{\tau}$. More specifically, let

$$
\hat{S}^{c}=\operatorname{span}\left\{\hat{\phi}_{1}, \cdots, \hat{\phi}_{l}\right\}, \quad l=\max \{K(i) \mid i=1, \cdots, M\} .
$$

Then $\hat{\phi}_{k}^{i} \in\left\{\hat{\phi}_{1}, \cdots, \hat{\phi}_{l}\right\}$. Since all basis functions of $\hat{S}^{c}$ vanish at the vertices of the reference element $\hat{\tau}$, the mapping

$$
\hat{v} \mapsto|\hat{v}|_{1, \hat{\tau}}=\left(\int_{\hat{\tau}} \nabla \hat{v} \cdot \nabla \hat{v} d \hat{x}\right)^{\frac{1}{2}}, \quad \hat{v} \in \hat{S}^{c}
$$

defines a norm in $\hat{S}^{c}$. Hence, the norms $\left(\sum_{k \in K(i)}\left|d_{k}^{i} \hat{\phi}_{k}^{i}\right|_{1, \hat{\tau}}^{2}\right)^{1 / 2}$ and $\left|\hat{v}_{i}\right|_{1, \hat{\tau}}$ are equivalent for the space $\hat{S}^{c}$ is finite-dimensional. Moreover, since the mapping $\theta_{\tau_{i}}$ is affine and meshes are regular, we also have another equivalence relation between the norms $\left|\tilde{v}_{i}\right|_{1, \tau_{i}}$ and $\left|\hat{v}_{i}\right|_{1, \hat{\tau}}$. All constants appear in each equivalence are independent of the mesh parameter $h$. We thus have the inequalities (2.15).

Observe that Eq. (2.9) can now be localized in each element $\tau_{i}$ if the test and trial functions on the left-hand side are taken to be the halved-functions of (2.12). On the other side, the residual term of (2.9) will involve the residual errors in each pair of adjacent elements $\tau_{i}$ and $\tau_{j}$ as well as the errors on the common edge if the conforming shape function $\phi_{i j}$ of (2.11) is kept as a test function for the residual term. This motivates us to modify the conforming problem (2.9) and consider the following problem: On each element $\tau_{i}$, find $\tilde{e}_{i} \in S_{i}^{c}$ such that

$$
B_{h}\left(\tilde{e}_{i}, \tilde{\phi}_{j}^{i}\right)=\frac{1}{2}\left(F\left(\phi_{i j}\right)-B\left(u_{h}, \phi_{i j}\right)\right) \quad \forall \tilde{\phi}_{j}^{i} \in S_{i}^{c},
$$

where the bilinear form $B_{h}$ is "nonconforming" and represents a restriction of $B$ to each element, i.e.,

$$
B_{h}\left(w_{i}, v_{i}\right)=\int_{\tau_{i}} \nabla w_{i} \cdot \nabla v_{i} d x
$$

for all $w_{i}$ and $v_{i}$ in $S_{i}^{c}$. The uniqueness and existence of $\tilde{e}_{i}$ is guaranteed since the bilinear form induces a norm in the space $S_{i}^{c}$ and the space itself is finite-dimensional. Let $e^{n}$ denote the sum of the local solutions, i.e., 


$$
e^{n}=\sum_{i=1}^{M} \tilde{e}_{i} \in S^{n}
$$

The norm of $e^{n}$

$$
\left\|e^{n}\right\|_{B_{h}}:=\left(\sum_{i=1}^{M}\left\|\tilde{e}_{i}\right\|_{B_{h}}^{2}\right)^{1 / 2}
$$

can then be used as a (nonconforming) error estimator for the approximate solution $u_{h}$ of (2.3). We now state the main result of the nonconforming error estimation.

Theorem 2. With the same assumptions as in Theorem 1, let $\tilde{e}_{i}$ be the solution of (2.16) and $e^{n}$ be defined by (2.17). Then

$$
(1-\rho) \sqrt{1-\gamma^{2}}\|e\|_{B} \leq\left\|e^{n}\right\|_{B_{h}} \leq C_{4}\|e\|_{B}
$$

where $C_{4}$ is a positive constant, $\rho$ and $\gamma$ are given in Theorem 1, and all the constants are independent of the mesh size $h$.

Proof. Let $e^{c} \in S^{c}$ be the solution of (2.9). By the definition of $S^{c}$ in (2.11), the solution can be written as

$$
e^{c}=\frac{1}{2} \sum_{i} \sum_{k \in K(i)} c_{i k} \phi_{i k}
$$

where $c_{i k}$ are midpoint nodal values of $e^{c}$ associated with the adjacent pair $\tau_{i}$ and $\tau_{k}$. Note that $c_{i k}=c_{k i}$ and $\phi_{i k}=\phi_{k i}$ for all $k \in K(i)$ and the summations will visit each element twice. From (2.9) and (2.16), we have

$$
\begin{aligned}
B_{h}\left(\tilde{e}_{i}, \tilde{\phi}_{k}^{i}\right) & =\frac{1}{2}\left(F\left(\phi_{i k}\right)-B\left(u_{h}, \phi_{i k}\right)\right) \\
& =\frac{1}{2} B\left(e^{c}, \phi_{i k}\right)
\end{aligned}
$$

for any fixed $i=1,2, \cdots, M$ and for all $k \in K(i)$. Multiplying both sides by $c_{i k}$ and then summing over the index set $K(i)$ yield

$$
B_{h}\left(\tilde{e}_{i}, \sum_{k \in K(i)} c_{i k} \tilde{\phi}_{k}^{i}\right)=B_{h}\left(\tilde{e}_{i},\left.e^{c}\right|_{\tau_{i}}\right)=\frac{1}{2} B\left(e^{c}, \sum_{k \in K(i)} c_{i k} \phi_{i k}\right) .
$$


It follows that

$$
\begin{aligned}
\left\|e^{c}\right\|_{B}^{2} & =B\left(e^{c}, e^{c}\right) \\
& =B\left(e^{c}, \frac{1}{2} \sum_{i} \sum_{k \in K(i)} c_{i k} \phi_{i k}\right) \\
& =\sum_{i} B_{h}\left(\tilde{e}_{i},\left.e^{c}\right|_{\tau_{i}}\right) \\
& \leq \sum_{i}\left\|\tilde{e}_{i}\right\|_{B_{h}}\left\|e^{c}\right\|_{B, \tau_{i}} \\
& \leq \frac{1}{2} \sum_{i}\left(\left\|\tilde{e}_{i}\right\|_{B_{h}}^{2}+\left\|e^{c}\right\|_{B, \tau_{i}}^{2}\right) \\
& =\frac{1}{2}\left(\left\|e^{n}\right\|_{B_{h}}^{2}+\left\|e^{c}\right\|_{B}^{2}\right) .
\end{aligned}
$$

Hence

$$
\left\|e^{c}\right\|_{B} \leq\left\|e^{n}\right\|_{B_{h}},
$$

which together with (2.10) proves the left inequality of (2.19). For the right inequality, we first extend $\tilde{e}_{i}=\sum_{k \in K(i)} d_{k}^{i} \tilde{\phi}_{k}^{i} \in S_{i}^{c}$ to a new function $e_{i}^{+}$ defined by

$$
e_{i}^{+}=\sum_{k \in K(i)} d_{k}^{i} \phi_{i k}
$$

which obviously has support on the extended subdomain

$$
T_{h}(i):=\tau_{i} \cup\left(\cup_{k \in K(i)} \tau_{k}\right)
$$

from the element $\tau_{i} \in T_{h}$. Note that $e_{i}^{+} \in S^{c}$ and

$$
\begin{aligned}
\left\|\tilde{e}_{i}\right\|_{B_{h}}^{2} & =B_{h}\left(\tilde{e}_{i}, \tilde{e}_{i}\right) \\
& =\frac{1}{2}\left(F\left(e_{i}^{+}\right)-B\left(u_{h}, e_{i}^{+}\right)\right) \\
& =\frac{1}{2} B\left(e^{c}, e_{i}^{+}\right) \\
& \leq \frac{1}{2}\left\|e^{c}\right\|_{B, T_{h}(i)}\left\|e_{i}^{+}\right\|_{B} .
\end{aligned}
$$

Since the shape regularity of $T_{h}$ implies the local quasi-uniformity of the mesh, the areas of the two adjacent elements $\tau_{i}$ and $\tau_{k}$ are comparable, that is, the ratio of the Jacobians of the two affine transformations $\theta_{\tau_{i}}$ and $\theta_{\tau_{k}}$ is bounded. Moreover, on the reference elements (triangle or rectangle), the ratio of $\left|\hat{\phi}_{k}^{i}\right|_{1, \hat{\tau}}$ and $\left|\hat{\phi}_{i}^{k}\right|_{1, \hat{\tau}}$ is either equal to one (both $\tau_{i}$ and $\tau_{k}$ are of the same type of elements) or fixed (one is triangle and the other is rectangle). Therefore, there 
is a positive constant $C_{5}$, which is independent of the elements $\tau_{i}$ and $\tau_{k}$, such that

$$
\left|\phi_{i k}\right|_{1, \tau_{k}}^{2} \leq C_{5}\left|\phi_{i k}\right|_{1, \tau_{i}}^{2}
$$

or equivalently

$$
\left\|d_{k}^{i} \phi_{i k}\right\|_{B, \tau_{k}}^{2} \leq C_{5}\left\|d_{k}^{i} \phi_{i k}\right\|_{B, \tau_{i}}^{2}
$$

Hence,

$$
\begin{aligned}
& \left\|e_{i}^{+}\right\|_{B}^{2}=\left\|e_{i}^{+}\right\|_{B, \tau_{i}}^{2}+\sum_{k \in K(i)}\left\|e_{i}^{+}\right\|_{B, \tau_{k}}^{2} \\
& =\left\|\tilde{e}_{i}\right\|_{B_{h}}^{2}+\sum_{k \in K(i)}\left\|d_{k}^{i} \phi_{i k}\right\|_{B, \tau_{k}}^{2} \\
& \leq\left\|\tilde{e}_{i}\right\|_{B_{h}}^{2}+C_{5} \sum_{k \in K(i)}\left\|d_{k}^{i} \phi_{i k}\right\|_{B, \tau_{i}}^{2} \\
& =\left\|\tilde{e}_{i}\right\|_{B_{h}}^{2}+C_{5} \sum_{k \in K(i)}\left\|d_{k}^{i} \tilde{\phi}_{k}^{i}\right\|_{B_{h}}^{2} \\
& \leq\left\|\tilde{e}_{i}\right\|_{B_{h}}^{2}+\frac{C_{5}}{C_{1}}\left\|\tilde{e}_{i}\right\|_{B_{h}}^{2} \\
& =C_{6}\left\|\tilde{e}_{i}\right\|_{B_{h}}^{2},
\end{aligned}
$$

where $C_{6}=1+\frac{C_{5}}{C_{1}}$. From $(2.18),(2.20)$, and (2.23), we have

$$
\begin{aligned}
\left\|e^{n}\right\|_{B_{h}}^{2} & =\sum_{i}\left\|\tilde{e}_{i}\right\|_{B_{h}}^{2} \\
& \leq \frac{C_{6}}{4} \sum_{i}\left\|e^{c}\right\|_{B, T_{h}(i)}^{2} \\
& \leq \frac{C_{6}}{4} \max _{i}\left\{\left|T_{h}(i)\right|\right\}\left\|e^{c}\right\|_{B}^{2} \\
& \leq \frac{5 C_{6}}{4}\left\|e^{c}\right\|_{B}^{2},
\end{aligned}
$$

where $\left|T_{h}(i)\right|$ denotes the number of elements in the subdomain, which is less than or equal to 5 (a rectangle having 4 adjacent elements at most). The right inequality of (2.19) then follows with $C_{4}=\frac{\sqrt{5 C_{6}}}{2}$ which is independent of $h$.

Remark. Obviously, the previous argument holds for more general finite element spaces $S$ and $S^{c}$ as long as the condition (2.5) is satisfied. Furthermore, if both conforming and nonconforming formulas, i.e., (2.9) and (2.16) are used, we obtain a generic method for all $h, p$, and $h p$ adaptivity. For 
example, assuming that the approximation order is $p$, if the next hierarchical shape functions of degree $p+1$ are internal modes [22], we then use (2.9) to compute the error estimator. Otherwise, we use (2.16) for side modes. Note that the estimators of [9] and [11] based on hierarchical bases are only conforming. Evidently, these estimators will fail for the Laplace equation with linear finite element approximation (see Example 4.1 in [15]). Of course for odd-order approximations, there are many other alternatives, for example, the Bank-Weiser estimator [10]. Nevertheless, we feel that formula (2.16) is a more generic extension of (2.9). This makes implementation of adaptive computations easier for more general applications and numerical methods as mentioned in the introduction. We refer particularly to [17] on how this generic feature can be fully exploited in the object-oriented programming.

\section{The Error Estimator for the General Problem}

We now apply the nonconforming error estimator to the more general selfadjoint problem

$$
\begin{aligned}
-\nabla \cdot a(x) \nabla u(x)+b(x) u(x) & =f(x) & & \text { in } \Omega, \\
u & =0 & & \text { on } \partial \Omega_{D}, \\
a(x) \frac{\partial u}{\partial n} & =g & & \text { on } \partial \Omega_{N}
\end{aligned}
$$

with $\Omega$ as in (2.1), $a(x)$ in $C^{1}(\bar{\Omega}), b(x)$ in $C^{0}(\bar{\Omega})$. We assume there exist constants $\underline{a}, \bar{a}, \underline{b}, \bar{b}$ such that $0<\underline{a} \leq a(x) \leq \bar{a}$, and $0 \leq \underline{b} \leq b(x) \leq \bar{b}$ for $x \in \bar{\Omega}$. The associated variational problem is to find $u \in H(\Omega)$ such that

$$
A(u, v)=F(v) \quad \forall v \in H(\Omega),
$$

where

$$
\begin{aligned}
H(\Omega) & :=\left\{u \in H^{1}(\Omega): u=0 \text { on } \partial \Omega_{D}\right\}, \\
A(u, v) & :=\int_{\Omega}(a \nabla u \cdot \nabla v+b u v) d x, \\
F(v) & :=\int_{\Omega} f v d x+\int_{\partial \Omega_{N}} g v d s .
\end{aligned}
$$

Corresponding to (2.3), the finite element solution $u_{h} \in S$ now satisfies

$$
A\left(u_{h}, v_{h}\right)=F\left(v_{h}\right) \quad \forall v_{h} \in S .
$$

The conforming error problem is then to determine $e^{c} \in S^{c}$ such that

$$
A\left(e^{c}, v\right)=F(v)-A\left(u_{h}, v\right) \quad \forall v \in S^{c} .
$$


The bilinear form $A(\cdot, \cdot)$ induces the $A$-norm $\|\cdot\|_{A}$. Assume that Assumption 1 holds with the $B$-norm replaced by the $A$-norm. Evidently, Theorem 1 is still valid with (2.10) replaced by

$$
(1-\rho) \sqrt{1-\gamma^{2}}\|e\|_{A} \leq\left\|e^{c}\right\|_{A} \leq\|e\|_{A}
$$

Using the Friedrichs inequality [4], it is well-known that the $A$ - and $B$-norms are equivalent, i.e., for any $v \in H(\Omega)$, we have

$$
C_{7}\|v\|_{B} \leq\|v\|_{A} \leq C_{8}\|v\|_{B},
$$

where $C_{7}=\underline{a}$ and $C_{8}$ is some positive constant.

The equivalence clearly suggests that it is simpler, in terms of implementation, to use the bilinear form $B(\cdot, \cdot)$ for the left-hand side of the nonconforming equation (2.16) than to use $A(\cdot, \cdot)$. The nonconforming problem for the general case is thus to determine, in each element $\tau_{i}, \tilde{e}_{i} \in S_{i}^{c}$ such that

$$
B_{h}\left(\tilde{e}_{i}, \tilde{\phi}_{j}^{i}\right)=\frac{1}{2}\left(F\left(\phi_{i j}\right)-A\left(u_{h}, \phi_{i j}\right)\right) \quad \forall \tilde{\phi}_{j}^{i} \in S_{i}^{c}
$$

The use of a simpler (Laplace) operator for the left-hand side of local residual problems was suggested in [3]. The result of the previous section remains valid for the general problem (3.1) as follows:

Theorem 3. Let $u \in H(\Omega), u_{h} \in S$ and $\tilde{e}_{i}$ be the solutions of (3.2), (3.3), and (3.7), respectively. Let $e=u-u_{h}$ and $e^{n}$ be defined by (2.17). If Assumption 1 holds then

$$
C_{9}\|e\|_{B} \leq\left\|e^{n}\right\|_{B_{h}} \leq C_{10}\|e\|_{B},
$$

where $C_{9}$ and $C_{10}$ are positive constants independent of the mesh size $h$.

Proof. The proof proceeds as that of Theorem 2. We use the same notation there, e.g., $c_{i k}, e_{i}^{+}$etc.. From (3.4) and (3.7), we have

$$
\begin{aligned}
B_{h}\left(\tilde{e}_{i}, \tilde{\phi}_{k}^{i}\right) & =\frac{1}{2}\left(F\left(\phi_{i k}\right)-A\left(u_{h}, \phi_{i k}\right)\right) \\
& =\frac{1}{2} A\left(e^{c}, \phi_{i k}\right)
\end{aligned}
$$


and hence, by (3.6),

$$
\begin{aligned}
C_{7}^{2}\left\|e^{c}\right\|_{B}^{2} & \leq\left\|e^{c}\right\|_{A}^{2} \\
& =A\left(e^{c}, e^{c}\right) \\
& =\sum_{i} \frac{1}{2} A\left(e^{c}, \sum_{k \in K(i)} c_{i k} \phi_{i k}\right) \\
& =\sum_{i} B_{h}\left(\tilde{e}_{i}, \sum_{k \in K(i)} c_{i k} \tilde{\phi}_{k}^{i}\right) \\
& =\sum_{i} B_{h}\left(\tilde{e}_{i},\left.e^{c}\right|_{\tau_{i}}\right) \\
& \leq \sum_{i}\left\|\tilde{e}_{i}\right\|_{B_{h}}\left\|e^{c}\right\|_{B, \tau_{i}} \\
& \leq \frac{1}{2} \sum_{i}\left(\frac{1}{\varepsilon}\left\|\tilde{e}_{i}\right\|_{B_{h}}^{2}+\varepsilon\left\|e^{c}\right\|_{B, \tau_{i}}^{2}\right) \\
& =\frac{1}{2}\left(\frac{1}{\varepsilon}\left\|e^{n}\right\|_{B_{h}}^{2}+\varepsilon\left\|e^{c}\right\|_{B}^{2}\right)
\end{aligned}
$$

for any positive number $\varepsilon$. The left inequality of (3.8) thus follows by choosing $\varepsilon=C_{7}^{2}$ and by using (3.5) and (3.6) with the constant $C_{9}=C_{7}^{3}(1-$ $\rho) \sqrt{1-\gamma^{2}} / C_{8}$. Corresponding to $(2.20)$, we have

$$
\begin{aligned}
\left\|\tilde{e}_{i}\right\|_{B_{h}}^{2} & =\frac{1}{2}\left(F\left(e_{i}^{+}\right)-A\left(u_{h}, e_{i}^{+}\right)\right) \\
& =\frac{1}{2} A\left(e^{c}, e_{i}^{+}\right) \\
& \leq \frac{1}{2}\left\|e^{c}\right\|_{A, T_{h}(i)}\left\|e_{i}^{+}\right\|_{A} \\
& \leq \frac{C_{8}}{2}\left\|e^{c}\right\|_{A, T_{h}(i)}\left\|e_{i}^{+}\right\|_{B} .
\end{aligned}
$$

Hence,

$$
\begin{aligned}
\left\|e^{n}\right\|_{B_{h}}^{2} & =\sum_{i}\left\|\tilde{e}_{i}\right\|_{B_{h}}^{2} \\
& \leq \frac{C_{6} C_{8}^{2}}{4} \sum_{i}\left\|e^{c}\right\|_{A, T_{h}(i)}^{2} \\
& \leq \frac{5 C_{6} C_{8}^{2}}{4}\left\|e^{c}\right\|_{A}^{2},
\end{aligned}
$$

which proves the right inequality of (3.8) with $C_{10}=C_{8}^{2} \sqrt{5 C_{6}} / 2$. 


\section{Numerical Example}

We consider Laplace's equation

$$
\begin{aligned}
\Delta u=0 & \text { in } \Omega=(-1,1) \times(0,1), \\
u=0 & \text { on } \partial \Omega_{D}, \\
\frac{\partial u}{\partial n}=g & \text { on } \partial \Omega_{N},
\end{aligned}
$$

where $\partial \Omega_{D}=\{(x, y): x \in[-1,1], y=0\}, \partial \Omega_{N}=\partial \Omega \backslash \partial \Omega_{D}$ and $g$ is defined so that, in polar coordinates, the exact solution $u$ becomes $u=c \cdot r^{1 / 2} \sin \frac{\alpha}{2}$, where $\mathrm{c}=0.0700754[7]$.

For our computations, the domain is partitioned by squares using the 1irregular refinement scheme [17] and bilinear elements are used to define $S$. The halved-functions (2.12) are constructed by using the following shape functions

$$
\begin{array}{ll}
\hat{\phi}_{1}(\xi, \eta)=\left(1-\xi^{2}\right)(1-\eta) / 2, & \hat{\phi}_{2}(\xi, \eta)=(1+\xi)\left(1-\eta^{2}\right) / 2, \\
\hat{\phi}_{3}(\xi, \eta)=\left(1-\xi^{2}\right)(1+\eta) / 2, & \hat{\phi}_{4}(\xi, \eta)=(1-\xi)\left(1-\eta^{2}\right) / 2,
\end{array}
$$

defined on the reference element $\hat{\tau}=\{(\xi, \eta):|\xi| \leq 1,|\eta| \leq 1\}$.

An adaptive process using the nonconforming estimator (2.18) begins with the initial mesh shown in Fig. 1 and ends with the final mesh shown in Fig. 2. The performance of the estimator is shown in Table I, where NN denotes the number of nodes.

This type of model problems has been used to test various estimators (see, e.g., $[1,7,10,20]$ ). As mentioned in [20], error estimators based on the solution of local residual problems give more accurate global error assessment as well as better local refinement indicators. This indeed has been observed from the numerical results of $[1,7,10,20]$ and this paper. The quality of our nonconforming estimator (the last column in Table I) coincides with that

Fig. 1. Initial mesh.

Fig. 2. Final mesh. 
TABLE I. Effectivity of the estimator

\begin{tabular}{ccc}
\hline \hline & $\|e\|_{B}$ & $\frac{\left\|e^{n}\right\|_{B_{h}}}{\|e\|_{B}}$ \\
\hline \multirow{2}{*}{$\begin{array}{cc} \\
6\end{array}$} & .0231 & 1.091 \\
15 & .0179 & 1.057 \\
37 & .0081 & 1.093 \\
68 & .0053 & 1.019 \\
165 & .0030 & 0.990 \\
412 & .0017 & 0.984 \\
816 & .0012 & 0.984 \\
1563 & .0008 & 0.986 \\
2215 & .0007 & 0.987 \\
3184 & .0005 & 0.988 \\
\hline \hline
\end{tabular}

of [1] in which the conforming estimator was first proposed for even-order finite element approximations. Finally, in comparison with the strong residual nonconforming estimator of [10], which involves the flux jumps, our estimator also improves in terms of effectiveness and implementation.

\section{ACKNOWLEDGMENT}

The authors would like to thank the referees for their helpful comments and suggestions on the manuscript.

\section{REFERENCES}

1. S. Adjerid and J. E. Flaherty, Second-order finite element approximations and a posteriori error estimation for two-dimensional parabolic systems, Numer. Math. 53 (1988), 183-198.

2. S. Adjerid, J. E. Flaherty and Y. J. Wang, A posteriori error estimation with finite element methods of lines for one-dimensional parabolic systems, Numer. Math. 65 (1993), 1-21.

3. M. Ainsworth and J. T. Oden, A unified approach to a posteriori error estimation using element residual methods, Numer. Math. 65 (1993), 23-50.

4. O. Axelsson and V. A. Barker, Finite Element Solution of Boundary Value Problems, Theory and Computation, Academic Press, 1984. 
5. I. Babuška and A. Miller, The post-processing approach in the finite element methods, Part 3: A posteriori error estimates and adaptive mesh selection, Internat. J. Numer. Methods. Engrg. 20 (1984), 2311-2324.

6. I. Babuška and W. C. Rheinboldt, Error estimates for adaptive finite element computations, SIAM J. Numer. Anal. 15 (1978), 736-754.

7. I. Babuška and D. Yu, Asymptotically exact a posteriori error estimator for bi-quadratic elements, Finite. Elem. Anal. Des. 3 (1987), 341-354.

8. R. E. Bank, Hierarchical bases and the finite element method, in: Acta Numerica 1996, A. Iserles, ed., Cambridge University Press, 1996, pp. 1-43.

9. R. E. Bank and R. K. Smith, A posteriori error estimates based on hierarchical bases, SIAM J. Numer. Anal. 30 (1993), 921-935.

10. R. E. Bank and A. Weiser, Some a posteriori error estimators for elliptic partial differential equations, Math. Comp. 44 (1985), 283-301.

11. F. A. Bornemann, B. Erdmann and R. Kornhuber, A posteriori error estimates for elliptic problems in two and three space dimensions, SIAM J. Numer. Anal. 33 (1996), 1188-1204.

12. V. Eijkhout and P. Vassilevski, The role of the strengthened Cauchy-BuniakowskiiSchwarz inequality in multilevel methods, SIAM Rev. 33 (1991), 405-419.

13. I.-J. Lin, D.-P. Chen and J.-L. Liu, Adaptive least squares finite element methods for the Stokes problem, J. Chinese Soc. Mech. Engrg. 18 (1997), 267-281

14. J.-L. Liu, Weak residual error estimates for symmetric positive systems, Numer. Funct. Anal. Optim. 14 (1993), 607-619.

15. J.-L. Liu, On weak residual error estimation, SIAM J. Sci. Comput. 17 (1996), 1249-1268.

16. J.-L. Liu, I.-J. Lin and M.-C. Hsieh, An outline of adaptive computation: theory, implementation, and applications, J. Chinese Soc. Mech. Engrg. 19 (1998), 149-157.

17. J.-L. Liu, I.-J. Lin, M.-Z. Shih, R.-C. Chen and M.-C. Hsieh, Object oriented programming of adaptive finite element and finite volume methods, Appl. Numer. Math. 21 (1996), 439-467.

18. J.-L. Liu and W. C. Rheinboldt, A posteriori finite element error estimators for indefinite elliptic boundary value problems, Numer. Funct. Anal. Optim. 15 (1994), 335-356.

19. J.-L. Liu and W. C. Rheinboldt, A posteriori finite element error estimators for parametrized nonlinear boundary value problems, Numer. Funct. Anal. Optim. 17 (1996), 605-637.

20. J. T. Oden, L. Demkowicz, W. Rachowicz and T. A. Westermann, Toward a universal $h-p$ adaptive finite element strategy, Part 2. A posteriori error estimation, Comput. Methods Appl. Mech. Engrg. 77 (1989), 113-180. 
21. J. T. Oden, W. Wu and M. Ainsworth, An a posteriori error estimate for finite elmenent approximations of the Navier-Stokes equations, Comput. Methods Appl. Mech. Engrg. 111 (1994), 185-202.

22. B. A. Sźabo and I. Babuška, Finite Element Analysis, Wiley, New York, 1991.

23. R. Verfürth, A Review of A Posteriori Error Estimation and Adaptive MeshRefinement Techniques, B. G. Teubner, Stuttgart, 1996.

24. O. C. Zienkiewicz and J. Z. Zhu, A simple error estimator and adaptive procedure for practical engineering analysis, Internat. J. Numer. Methods. Engrg. 24 (1987), 337-357.

Department of Applied Mathematics, National Chiao Tung University

Hsinchu, Taiwan

E-mail: jinnliu@math.nctu.edu.tw. 